\title{
Tips for effective English writing
}

\section{Effective English writing: A guide}

\section{Dr. Gareth Dyke ${ }^{a}$}

aUniversity of Debrecen, Hungary. Email: gareth.dyke@ vocs.unideb.hu

How to write English more effectively and easily? The goal of this guide is to help you write effectively in English. We know, it's not easy! Especially if English is your second, third, or fourth language. We are here to help. We'll consider cognition, how to write effectively, and how to increase the resultant visibility of your research. We'll cover some rules and guidelines as well as tips and tricks. Writing effectively in English is all about learning to be an effective communicator. It's not about grammar, it's not about learning all the rules. Writing is a skill you can develop, not a talent. This is important to keep in mind as we get started.

This guide is structured into four parts to keep things simple. First, we'll consider the logical structure of an academic paper, before moving on to help you improve your readability. We then look at the use of proper tenses in English academic writing before wrapping up with some discussion of writing skills (not talents, remember!). Our goal with these structured easy-to-follow guides is to help you increase your chance of publication and your research impact.

1. Effective academic writing:
Understanding your reader

We don't write academic papers so we can pin them to the wall, or give them to our mothers as presents. No. We write academic research articles so others will read them and, hopefully, cite them. This is our goal: Writing a paper that does not get read and cited is almost as bad as not writing a paper at all. Readers are key. We must appeal to our readers as academic writers. We must engage and capture their interest and do the best we can to make sure readers get to the end of our work and think: I agree! I do think that this is the correct, expected outcome. More on this later.
Here are some fun facts about your readers who, by the way, are almost certainly not going to be native English speakers. Where is most of the research in the world getting done these days? Much of it is taking place in Asia, in countries like Japan and China where English is not spoken as a first language. It's also informative to think about why we choose to share our research. What's the purpose of an academic paper? Why write at all? The goal of writing a research article is to explain new information to readers in order that they will remember and use that content. You need to get your message across. So, your goals as a writer are to explain new information in such a way that readers will remember, retain, and use your new knowledge.

The goal of this guide is to help you do this easily and effectively in English. We know that English is probably not your native language, but even if it is: We are here to help!

\section{Cognitive learning: The basis for effective communication}

Effective writers are able to understand their readers by applying three important learning principles. We'lll deal with each of these in turn: cognitive load theory, cognitive bias, and reader expectations. The first, cognitive load theory, has to do with understanding just how much new information your readers are able to process, while the second, cognitive bias, warns us not to assume that our readers know as much as we do (we'll come back to this). Finally, the logical presentation of information for readers is important: the readers of academic papers expect to see and absorb information in a certain specific order. This is the logical structure of an academic article.

2.1 Cognitive load theory: How much information can our readers process? 
Did you know that research has shown that the human brain can only process between seven and nine new pieces of information at any time? This is cognitive load. This feeds into our understanding of sentence structure and length. It's best therefore to write using sentences that are shorter rather than longer: sentences 10 words in length tend to be recalled by readers $98 \%$ of the time, while those that are 20 words in length are recalled $85 \%$ of the time. The drop-off after that is significant: sentences 30 words in length are recalled $70 \%$ of the time, while those 50 words in length have just $58 \%$ recall.

What does this mean? We recommend that you aim for 10-20 words per sentence on average. Some longer and some shorter are still fine of course, but as a general rule: shorter sentences are better than longer cumbersome ones. At the same time, aim for just one idea per sentence. This is the concept of topic sentences (which we'll return to a little later on in this guide). Use semi-colons in sentences only when necessary and make sure you clearly and throughly explain your ideas to your readers.

Cognitive load theory, the amount of information readers are able to process, also teaches us to avoid the use of unnecessary words that do not add value to ideas. Here's a simple rule to follow in your writing: Can you delete a word without changing the meaning of a sentence? If yes, then delete those words. The best way to manage this is to read out your work aloud: read to your pets, read to your dog, read to your cat, read to your goldfish! Reading your work aloud helps to identify those cumbersome phrases and words that are not needed for meaning, words that do not add values to a presented idea.

Another great trick is to become friends with adverbs. These are an editor's best friends! Adverbs are great because their shorten sentences and augment meaning.

\subsection{Cognitive bias: Understanding your reader}

Cognitive bias is where you assume that your readers know the same amount about a particular subject as you do, the expert researcher. Sounds obvious, but you'd be amazed how many academic papers do just that: confuse the reader by assuming a level of knowledge that's just not there. It's like my nephew: when I visit him, he is invariably playing computer games. I'm a little older. But he talks to me as though I really do know what he's talking about: cognitive bias in action. Make sure to take the time to carefully explain things in your papers so that your readers don't lose interest.

It's important to remember that your interests boil down to a small area within a much larger field of research. Science is vast and difficult. Never assume your readers know as much as you do about your work. Provide helpful background information so that your readers are able to understand your new, important, information.

As editors, we encounter cognitive bias throughout academic articles. In the Introduction, writers don't define their ideas of theories effectively and don't tell readers about their research problem. What's the issue that your paper seeks to address? Similarly, in the Methods, writers don't define their methodologies or the significance of their data. How was the study conducted? What was done? How can the work be repeated? Conclusions too; writers don't discuss the implications of their research or possible future directions. Which new hypotheses or ideas stem from your project? Talk about these ideas in your discussion and conclusions so that other researchers will be forced to cite you. They will have to use your work: Don't just let others come up with ideas on their own on the basis of your work! This is also key, by the way, as a strategy to get your work successfully through peer review.

Don't be ambiguous in your writing: As we've discussed, it's important to clearly explain your ideas to your readers. With this in mind, try to avoid the use of unclear subjects in your writing: words like 'this', 'that', 'these', 'those', 'they', and 'it' can be confusing for readers.

‘.. 320 and 531 participants were randomly allocated into experimental and control groups. They were they ...'

This is confusing. Which group? What was the treatment? This is an example of cognitive bias: You know what you mean (of course, you are the subject area specialist, the expert, but does your reader? Perhaps not). In this example we might rather write "The participants were ...." to avoid any issues in understanding.

In a similar vein, lets talk about the issue of qualitative words. Words 'some', 'most', and 'few'. You are a quantitative researchers, you 
deal in absolutes: there is no place for these words in your papers. 'Few of the participants experienced an issue in treatment' What does this mean? What does 'few' refer to here? Are we talking about $10 \%, 5 \%, 1 \%$..? This is just another example of where, and why, it's important to clearly explain your ideas to your readers to avoid a loss of understanding. Better to write: ' 10 participants experienced adverse events out of a total sample of 2,000'.

Subjective words are also an issue in this context. These are superlative words, informal words like 'surprisingly', 'strikingly', and 'interestingly'. Again, academic writing should be objective, not subjective and so, please, have a think about whether your result was really so 'surprising' or 'interesting'. The use of these words can imply a lack of knowledge, at least to readers and peer reviewers.

One of my favourite subjective words, is 'unexpectedly'. Although sometimes good to use as a linking word, it's nevertheless important to think about meaning as often words like this are used at the start of sentences but could just be deleted. 'Unexpectedly, the results of this study imply ...' would be better just written as 'The results of this study imply ...'. Simpler and to the point.

I once worked with a French researcher who started lots of his English sentences with 'Be that as it may, ...'. Again. Subjective and not needed. As we've discussed, read your work aloud and you'll be able to much more easily highlight these kinds of issues with word choice and word use.

\subsection{Logical information presentation}

If you are interested in learning more about the shape of academic papers, especially ones in STM subjects, then check out our other guidebook in this series, our 'Clinical Research Writing Template'. In this companion guide, we talk in detail about the shape of research articles and provide templates for effective (and easier) academic writing. Here, we'll talk more about effective communication in English.

Effective communicators are effective guides, leading their readers through their articles from one idea to the next. This is what you are aiming for with your articles. You want your readers to get to the end of your articles and think: 'I agree, I expected that'. 'I think the paper was well-written and engaging', and lets face it: if people don't find your papers interesting, well-written, and engaging they'll probably turn off after the Title and Abstract and go off and do something else.

Academic papers are no different from any other kind of creative writing, such as detective stories or film plots. Readers need to be kept engaged and they need to know where they have come from and where they are going. Structure is key to creating a road map for readers as well as the use of signposting words and phrases. The use of these kinds of linking words and phrases help readers to more quickly understand expected ideas.

The logical structure of sentences builds into the structure of paragraphs, as we'll see. Let's have a think about sentences to start with; one good idea to think about to get us started is to place the topic at the start and then the stress position, the most impotant message, at the end of your sentence in order to link once sentence into the next one. This stress position at the end of a sentence links into the next, as well as giving readers the topic of the next. This is an important rule of thumb for sentence writing in general: flow from one into another with key information linking into the next sentence there at the end. This technique is called 'signposting', and it also works at the level of paragraphs (building on this simple approach for sentences).

In this regard, think about logically structuring your writing to get your message across to readers by introducing an idea, then developing it, and then going onto expanding on the importance of this theme. The end of each section then links into the next: readers will know what the next phrase or paragraph is about. This simple approach enables you to more effectively structure your writing around ideas. These are themes, topics for sentences. Keep your readers engaged with a logical writing structure.

\section{Logical structure to writing}

Clearly, the structure of sentences can influence your reader's interpretations. Getting this right is tricky, however, and includes word placement, clause length, and paragraph structure. We'll deal with each of these topics in turn and provide some insights into effective writing.

\subsection{Word placement}

Verbs are movement words. Action words. Words that do something. Readers expect verbs to closely follow the subject in a sentence, or 
they tend to get confused. Thus, a sentence with a subject placed earlier in the word order and separated far away from a verb tends to be harder to understand than one where the subject and verb are placed much closer together.

Let's consider an example:

'The viral infection over five years as measured in our data had declined greatly within the Japanese population'

'The viral infection had declined greatly over five years within the Japanese population'

General rule: try to keep the subject and action towards the beginning of a sentence in order to aid understanding for readers.

Words at the beginning of a sentence do tend to carry more weight than those in the middle. In addition to subjects and actions, important words should also therefore be placed as early as possible.

'The conversion from one kind of engine to another, however, was more frequent after initial testing'.

Compared to:

'However, the conversion from one kind of engine to another was more frequent after initial testing'.

The second sentence is the easier to read.

Similarly, it is also important to place negative words towards the start of sentences, again, in order to get your message across. Consider one example:

'We found evidence supporting the role of social programs in increasing wages was lacking'.

Or

'We found no evidence supporting the role of social programs in increasing wages.'

\subsection{Clause length}

Sounds complex. Do you know the difference between a so-called 'main' clause and a 'subordinate' one? A main clause is a sentence, or part of a sentence, that contains a subject and an object while a subordinate clause is a sentence fragment that does not make sense alone. Subordinate clauses can be short, one word like 'but' or 'however', or longer - like my personal favourite, 'but be that as it may'.

Knowing a writing trick involving clause length in this context is useful because, as we have discussed, sentence structure influences reader's interpretations. What this means in practice is that you can use the position of clauses to influence interpretations.

How about this example (imagine you are a peer-reviewer of a paper):

'Although this paper is important, there are a number of issues including in the methods'

'Although this paper is important, there are a number of issues including in the methods, the results, the data presentation, and in the figures'.

Which of these sentences comes across are more negative?

Adding clauses to a sentence can be used to reinforce a point or to ensure that your opinion is effectively communicated. What will the editor think when she/he reads this? If you write an opinion but all additional clauses to reinforce that point-of-view then your message gets across more clearly to your readers. This is a good one for peer-review, as we mentioned.

\subsection{Paragraph structure}

The structure of academic writing is important if you are going to be effective because science is vast. Your own specific subject area is just a small point in the wider universe of science and so it's key, as we've discussed, to work on techniques you can apply in your writing to get your message across as clearly and effectively as possible. This means that we have to act as guides when writing academic papers to ensure that our readers are brought into a broad field and then led to the narrow topic of your research.

What this means in practice is that it is a bad idea to start of your article writing with the specifics of your own research question. Not too many people will care about that from the outset. First you have to tell your reader why your work is important in a broader context, as we've already discussed in this guide. 
Start of your articles in the Introduction then by being broad: talk about the wider question, the bigger picture. Imagine: you are an ecologist working on heavy metal pollution in soil. Don't start your paper with the specific details of your question, start by broadening the scope and telling your reader who your work fits into a bigger picture:

'Soil pollution is important globally $\rightarrow$ Within the soil heavy metals accumulate $\rightarrow$ The aim of this paper is to ...'. Structure like this can work well.

This is the reason that academic papers are often described as being 'hour glass' (or egg-timer) shaped.

\section{Enhancing readability: Tips for success}

English writing style and structure influences readership and thus how many colleagues will use (and cite) your next paper. Effective English writing is a skill not a talent: Our guide aims to help you to become more efficient. In this section, we'll discuss the use of the active and passive voices in academic writing (perhaps the most often discussed and debated aspect of English technical writing) as well as the use (and abuse) of strong verbs. We'll also highlight a number of common mistakes that writers tend to make in English. Understand to avoid: If you know about the common errors we often encounter in the papers sent to us for editing then you can avoid these in your own writing!

\subsection{Active versus passive voice}

It's definitely the case that most people were taught in school to write up their experiments, where possible, in the passive voice. I remember this from my school days as well: 'the beaker was filled with water and placed on the bunsen burner', that sort of thing. The passive voice is where a subject is the recipient of a verb's action, while the active voice is the opposite: a subject acts upon it's verb. Put simply: 'I filled a beaker with water and placed it on the bunsen burner'. Which of these two sentences is easier to understand, keeping in mind that most of your readers are not going to be native English speakers?

It is clear that sentences written in the active voice are simpler, more direct, and easier to read and follow.

Consider another passive voice example: 'The models comparing the economic growth of East
Asia were evaluated'. It's just not too clear what was being evaluated and who was doing the evaluating. Indeed, people often remark that, since they remember being taught to use the passive voice when they were at school, then preferred use of the active voice in academic writing must be a new phenomenon. Not so. Actually, the use of active voice has been around for almost as long as academic publications: Charles Darwin wrote his paper is the 1840 s in the active voice, while the 1953 Nature paper 'Molecular structure of nucleic acids' by Watson and Crick was also written in this style .... 'We wish to suggest a structure for the salt of DNA ...'. Active voice writing is not new.

Indeed, it is the case that most (if not all) style guides used by leading international journals not that use of the active voice is preferred.

Are you convinced yet? A range of cognitive studies have actually show that both comprehension and readability are enhanced for active voice sentences, while sentences written in the passive require for brain power to understand. We recommend that, where possible, academic articles are written in the active (first person) voice wherever possible. Have a look at articles in Nature and Science if you need further convincing: articles in these leading journals (where we all aspire to publish our work) always have a phrase at the end of the Introduction which says 'In this paper, we show ...', or 'Here we show ...'. Words to that effect.

Should you always use the active voice? No. In a limited number of situations, use of the passive voice will be very useful and effective. In places where you want to avoid repetition and to define relationships which otherwise might be unclear as well as in the Methods section. It's also useful to use a switch from active to passive voice when you want to paraphrase text from another paper into one of yours and avoid plagiarism.

\subsection{Use strong verbs and avoid complex words}

Verbs are movement words, action words. Thus, to be as effective as possible it's important to use verbs in their pure, strong form. Lots of writers perform a process called nominalisation with their verbs, something which should be avoided if at all possible.

As you can see from the simple examples we provide its better to use less words and keep 
verbs in their strong original form (i.e., 'decided' rather than 'We made a decision' (active) or 'A decision was made' (passive) because this is more effective and you actually end up using less words.

What about the use of complex words? Lots of researchers assume that using complex words will make their work more credible and them, by extension, more intelligent looking. Wrong! Your readers have not swallowed a thesaurus and, of course, are often not native English speakers. Should I use complex words to make my writing look more impressive? No. Use simple language. Your ideas and findings are complex already; you are faced with the task of explaining these complex ideas to your reader.

\subsection{Some common English academic writing mistakes}

As we edit hundreds of academic articles each day, we've encountered most of the mistakes that authors tend to make. A number of the most common are summarised here: Not an exhaustive list, but nevertheless some ideas for your own writing. Why make the same mistakes that others have made in the past? Understand to avoid.

1. Making comparisons: The use of 'compared with' and 'compared to'.

The first of these, 'compared with' is used or comparing similar things, while the second 'compared to' is used for comparing things which are different. This is an important distinction; it's important to consider whether things you are comparing in your article are similar or different.

'My dog is big compared with my other dog'

'My dog is big compared to my cat'

It's a suble difference but nevertheless important to avoid confusion.

2. The use of 'can' and 'could': Used to identify something which has not yet been confirmed.

It's important to be careful with the use of these words in academic writing as they imply something which is not yet known. Something which is not yet confirmed.

'The over-expression of $\mathrm{xxx}$ can promote tumor progression'
This usage means that your compound, xxx, might promote tumor but that this is not yet known completely. Don't use can and could therefore to talk about the results of empirical quantitative studies: You have the evidence to support your claim.

'The over-expression of $\mathrm{xxx}$ promotes tumour progression'

Be confident. Be definitive. You have the results. Otherwise you would not be writing the paper.

3. The use of numbers up to nine.

Did you know that by convention numbers from one to nine are always written out in full form in academic papers? Numerals are used for those 10 and above. So, you'd write 'I collected eight different samples from 10 sites in this analysis'.

One exception to this rule, however, is when units are needed: Always write $8 \mathrm{~mL}$ or $8 \mathrm{~kg}$ (note the space between the number and units, this is also convention: Exceptions to this are ${ }^{\circ} \mathrm{C}$ and $\%-40^{\circ} \mathrm{C}$ and $40 \%$, for example).

Wait, it gets more complex: What about if you want to start a sentence with numbers and a unit? ' $33 \mathrm{~mL}$ of the solution were added ...' would be incorrect: You'd need to write 'Thirty-three milliliters of the solution were added ....'. Seems a little over the top, so why not switch to the active voice? (another good reason to use this form); then you could write 'We added $33 \mathrm{~mL}$ of the solution ...'. Simple.

\section{American English versus British English} spelling conventions

There are a good number of differences between American and British English in terms of spelling conventions and sentence formation. One of the most common mistakes we see in our editing work is a mixture of the two in one paper. Check your journal instructions for authors: Often journals specify one spelling convention or they just note that the convention should be consistent. Set your spell checker and ensure you don't mix up the two forms of English within one document.

Did you know, for example, that there are two spellings for the color/colour gray/grey (America/British)? Although you can always set the spelling correction function in your word processing software to either convention it's well-worth proof-reading before you submit 
and check the guidelines of the journal you are planning on submitting too: many journals don't care which spelling convention you use so long as you keep it consistent (don't mix the two kinds of spelling in one article, one very common issue that editors hate: 'analyze the colour', for the example), while others specify American or British conventions in their 'guidelines for authors'. An editor will quickly be able to spot if you've quickly re-worked a paper that was recently rejected from another outlet and then resubmitted it to them; put some time into this to maximise/maximize your chances of success!

5. The use of redundant and inappropriate words

It's tempting to write more than you need to in a sentence. This can be avoided: Shorter sentences are always better in English academic writing. As we discussed earlier, short sentences are better for comprehensive. Reduce the use of redundant words.

Some common examples include:

- $\quad$ small in size

- rectangular in shape

- blue in colour

- tenuous in nature

The use of inappropriate words just refers to usage that might sound strange to a native English speaker when applied in technical scientific writing. We recently edited a paper that was written by a leading Chinese medical doctor which included the phrase 'the government should not let the grass grow under it's feet when it comes to dealing with the gathering national crisis in antibiotic resistance. Patients who are resistant to antibiotics are springing up like mushrooms all over the country'. I've highlighted the phrases here that seem odd to a native speaker when included within academic writing. These usually come from translation or the use of online searches to find phrases that match with usage in a writer's native language: another good reason why, as we mentioned above, it's a good idea to try to think in English when writing in English. Words like 'amazing', 'marvellous', and 'fantastic', in English socalled superlatives, are often also encountered in the writings of ESL authors: 'the fantastic potential of land use changes in China' and 'a marvellous new application for digital elevation models' are two recent examples from our experience.

\section{Verb tense: Which, and where, in my paper?}

English verb tense use can be extremely confusing. Present perfect. Present simple? What's the difference. You might have taken lessons in English grammar, but, again, one of the key messages of this guide is: Remember your readers. Users of your papers are experiencing your work in real time. They are reading in the moment. This means that verb use in academic writing can actually be quite simple.

Simple present for most of your paper, if possible. Introduction, Results, and Discussion. The Methods? Well, these happened in the past, so simple past is good for that part. Keep it simple. Make your results the focus of your manuscript: Use simple present as the main tense.

What about the use of other tenses? Your use of future tense will be limited but it could appear towards the end of the Discussion where you talk about 'future' work, 'future' directions and other areas of thought leadership.

How do other authors manage this? A great tip is to check out a handful of papers in your own field that have appeared recently in high impact factor journals that you think are well-written and easy to read. Don't copy, but you can base your style on the work of others: how are the papers that you find easy to read and follow written? Do they use the first person active tense? (I bet they do). This also works well for developing your figures and tables: how do other successful authors put these together in their papers? What sorts of styles, softwares, colours, and formatting do they use?

\section{In sum: What sorts of writing habits do successful researchers have?}

This is a very good question, with no straightforward basic 'one-size-fits-all' answer. We've talked about writing effectively in English, one step towards being successful as a researcher. Colleagues who are 'successful' come in all shapes and sizes, of course; so, the first question to ask is 'what makes a successful researcher?' 'how do we measure success?'

Most people define a 'successful researcher' as someone who publishes lots of papers, wins substantial grant funding, and has developed a stable and productive career (or has the potential too). How can you maximise your 
effectiveness as a writer to ensure you are as productive as possible?

Well, the first and perhaps most important tip is: write as much as possible and write everything down. Note down ideas to develop later, even just fragments of text, and never throw anything away. I've found over the course of my career that documents written months, sometimes years, before can later prove very useful when I return to work on the same, or similar, topics. Organise files on your computer according to themes or questions and perhaps consider using some filing software (or just excel) to keep track of what you have already written in certain areas to you can easily return to these documents later.

Effective writing is difficult in any language, but especially so in your second (or third). One key thing to keep in mind when starting to write, to put your ideas down on paper (or on your computer screen) is that it's much more effective to think and write in English (if your goal is creating an English document) than to think and write in another language (Chinese) and then translate later. This is because information and structure will be lost in translation: We recommend that if you have the confidence to think and write in English then preferrably try this when putting documents together, but if you can't - if you feel that you just aren't at that level yet with your written English - then find a translation service that you trust, that has experience working with technical documents in your field.

Very many successful academic researchers don't actually start the writing process until they know what kind of paper they are doing to be putting together: which journal they will be submitting their work too. We teach in our workshops and training courses that 'target audience' (=target journal) is one of the three key things you should know before you start to write; the other two are 'message' and 'structure'.

Dealing with the second of these two first, it's absolutely key to know what the structure of the document you are writing is going to be before you start to write it; definitely one writing skill that successful academics have developed. Obviously, the kind of paper you'd write for a local interest journal will be quite different to the kind of paper you'd put together for Nature, Science, or another high impact factor international outlet.
So, in terms of key writing skills for successful researchers: try to think and write your first drafts in English if you can (and if you can't: find a translation service you trust to accurately parse your writing without loss of meaning), and be sure that you know your 'key message'as well as the 'target audience' and 'structure' of your writing before you get started.

Successful researchers tend to have develop a style to their academic writing; their papers read in similar ways. The best way to develop such a style that works for you in your written work is to base your early papers on others in your field that you admire and that you feel have been well-presented. Don't copy (I'm not advocating that) but take a look at the writing style and structure of some papers in your field that you think are well-written; what sorts of titles do they have? How are their abstracts stuctured? How do the introductions and discussion start and end? How are the methods sections presented? Those sorts of stylistic questions. If a certain kind of style has proved successful for other international colleagues, then it can also work well for you.

Decide on a voice in your writing that also works for you. Addressing this issue is actually one of the most common questions that we are asked in our author workshops: 'should I use passive, third person when writing my papers in English, or is the active, first person better? I've seen both used in academic articles'.

This is an interesting topic and actually quite debated in academic writing and teaching circles. Most writing courses will teach (and most colleagues feel that they have been taught) that it's good practice to use a passive, third person voice when writing up academic research work ('an experiment was performed', 'the following methods were used in this study', 'reagents were added to the PCR mix before further cycling was performed'). It's true: I was also taught this style when I was at school. However, an increasing body of literature is arriving at the consensus that actually use of an active, first person voice in academic writing is the better and more effective way to communication and keep a reader engaged. First person writing is simply easier to follow ('we performed an experiment', 'we used the following methods in this study', and 'we added reagents to the PCR mix and performed further cycling') and, thus, easier and more enjoyable to read.

Again, our advice is to have a look at some recently published papers in your field that you 
think are well-written and that have been widely cited and see what voice is used. Go for active, first person writing in your papers if possible and, above all, be consistent: one of the most common mistakes that non-native speakers make in their written English academic articles is to jump between active and passive voices in the same paper. They might start off in the introduction, for example, writing in the active voice ('we did this', 'I did that') but then switch to passive when putting together the methods section ('a reaction was performed', 'the following chemicals were added to the mixture'). This is one key thing to try to avoid in your written work.

Effective English writing comprises short sentences with limited punctuation. Try to write in the same way that you think and document as many of your ideas as possible. Writing down as much and as often as possible is one key skill that successful researchers tend to have developed, in my experience. Bear in mind that putting an academic paper together is a cumulative process, building your written work block-by-block, piece-by-piece; even the most successful, fluent native speaking writers can't sit down and just write a paper from start to finish. You can, however, make this process much easier by having a structure in mind and by keeping track of pieces of written work that you can use later for the various sections. If you sit and have a think about this process when you next have to write an academic article you might be pleasantly surprised by how much, say, of the introduction or methods section you already have in various forms already saved on your computer. Successful academic writers reuse, they re-cycle and re-formulate: building new pieces of work from building blocks they already have in their portfolio.

Publisher's note: Eurasia Academic Publishing Group (EAPG) remains neutral with regard to jurisdictional claims in published maps and institutional affiliations.

Open Access This article is licensed under a Creative Commons Attribution-NoDerivatives 4.0 International (CC BY-ND 4.0) licence, which permits copy and redistribute the material in any medium or format for any purpose, even commercially. The licensor cannot revoke these freedoms as long as you follow the licence terms. Under the following terms you must give appropriate credit, provide a link to the license, and indicate if changes were made. You may do so in any reasonable manner, but not in any way that suggests the licensor endorsed you or your use. If you remix, transform, or build upon the material, you may not distribute the modified material.

To view a copy of this license, visit https://creativecommons.org/licenses/by-nd/4.0/. 